\title{
SEMIÓTICA DEL TEXTO: NIVELES Y PASARELAS
}

\author{
Robert Marty
}

(Universidad de Perpignan)

\section{INTRODUCCIÓN}

La semiótica de Peirce de la cual se tratará aquí no se elaboró con el fin específico de analizar los textos bajo los aspectos de la llamada lingüística textual, al contrario de la narratología de A. J. Greimas y de la Escuela de París. Se encuentran en Peirce muchos ejemplos tomados de las lenguas, pero la mayor parte, por no decir todos, son relativos al funcionamiento de las palabras en cuanto a los objetos que representan o designan, o al papel lógico que desempeñan las proposiciones y los argumentos fuera de cualquier contexto. Pero eso no significa que una teoría de la narración o más generalmente del texto basada en la semiótica de Peirce no sea posible. Simplemente hay que constatar que, utilizando esta semiótica en el estado de desarrollo en el cual se encuentra en los manuscritos de Peirce, no se puede aún elaborar.algo que merezca el título de «análisis textual». Sin embargo, en los mismos manuscritos, encontramos algunas indicaciones y una lógica interna de desarrollo, que muestran cuál es el buen camino. Cuando Peirce escribe por un lado que «to- 
das las palabras, frases, libros y otros signos convencionales son símbolos» (2-292) y por otro lado que, considerando que la frase «Caín mató a Abel» tiene tres objetos (Abel, Caín y el asesinato), escribe «Pero el conjunto de los objetos puede ser considerado como formando un objeto complejo. En lo que sigue y a menudo en otras partes, haremos como si los signos tuvieran cada uno un objeto único a fin de ordenar por series las dificultades» (Peirce 2-230), vemos que contamos con toda la combinatoria que con signos sencillos, elementales produce signos tan complejos como un libro entero. Es el clásico y actual problema de la complejidad. Para resolverlo tendremos que traspasar las tradicionales taxinomías de los signos para introducir en la teoría de un sistema de relaciones de acuerdo con los principios fundadores y que permiten la descripción de esta complejidad. Esto no será difícil dado el carácter ante todo relacional de la fenomenología y finalmente de la filosofía entera de Peirce, quien escribe en el manuscrito 908 que «en toda clase de investigaciones, las divisiones más importantes son divisiones según la forma y no según las cualidades de la materia». Hablar de forma - y solamente de forma- es hablar de relaciones, exclusivamente. Por eso la teoría semiótica de Peirce no puede desarrollarse y profundizarse sin hundirla aún más en el álgebra de las relaciones. Por estos motivos haremos una breve presentación, adecuada a este propósito, de la semiótica de Peirce y de los prolongamientos que autoriza su formalización algebraica antes de aplicarla al análisis textual. Somos conscientes de que, en este estado primitivo de desarrollo, los resultados no pueden ser sumamente convincentes. Sin embargo, el éxito de la división de los signos en icono, índice y símbolo en los estudios literarios permite confiar en la capacidad de esta semiótica para incrementar el saber de estos estudios.

\section{MÁS ALLÁ DE LAS TAXINOMÍAS}

\section{La semiótica de Peirce es una semiótica general}

Para algunos, entre los cuales encontramos a Umberto Eco, una semiótica general puede ser solamente comparativa en su enfoque, es decir que tiene que contestar a la pregunta siguiente: ¿Revela la comparación de los sistemas diferentes de signos leyes comunes y sistemáticas permitiendo explicar de manera unificada su modo de funcionamiento? Para Peirce, «la semiótica es una ciencia de observación como cualquier ciencia positiva»y, por consiguiente, no se puede concebir que una semiótica sea otra cosa que una semiótica general porque será la ciencia de todos estos observables que son los fenómenos semióticos. 
Recordemos que el propio F. de Saussure decía en su Curso que la lingüística sólo es parte de esta ciencia general (la semiología) y que las leyes que averiguará serán aplicables a la lingüística. Así vemos que, según los primeros son las semióticas específicas las que deben constituir una semiótica general, mientras que los peirceanos piensan rigurosamente lo contrario. Otro enfoque epistemológico es el de la Escuela de París para la cual el signo es un objeto construido; pero como el modelo de esta construcción se toma en el lenguaje considerado como un universo cerrado, eso es equivalente a instituir una semiótica específica - por cierto muy importante- en semiótica general, matriz «a priori» de todos los fenómenos de significación.

\section{La semiótica de Peirce es una semiótica triádica}

2.1. Recordemos que una tríada es la unión de tres cosas en una, no en una cuarta, sino que es la unión de dos cosas en una tercera distinta de cada una de ellas. En una tríada hay tres elementos distintos pero cada uno de ellos actúa como elemento y también como relación entre los dos otros. En torno a esta concepción se anudan todas las dificultades y todo el interés de esta semiótica.

2.2. Peirce dio numerosas definiciones del signo; hemos recogido (Marty 1990: 367-384) 76 textos definiendo más o menos el signo. Todas son triádicas. Se pueden distinguir las definiciones «triádicas globales» (antes de 1905) en las cuales se trata de una relación triádica establecida globalmente entre signo, objeto e interpretante y las definiciones «triádicas analíticas» (después de 1905) en las cuales el establecimiento de la relación triádica se analiza en términos de determinaciones internas, a saber determinación del signo por el objeto seguida de la determinación del interpretante por el signo. Un ejemplo típico de la primera clase de definiciones es la siguiente de 1903: «Un representamen (o signo) es el sujeto de una relación triádica con un segundo llamado su objeto, para un tercero, llamado su interpretante» (Peirce 1-541). Un ejemplo típico de la segunda clase de definiciones es la siguiente de 1908: «Un signo es, por un lado, en cierto modo determinado por un objeto, y, por otro lado, determina cierto estado de conciencia (awareness), y eso de tal manera que este estado de conciencia es así determinado por este objeto» (Peirce MS 612).

2.3. Hay que señalar aquí un error desgraciadamente a menudo cometido en la representación de la tríada por un diagrama. En efecto muchos autores representan la tríada por un triángulo; nunca Peirce lo hizo porque esta representación corresponde a una tríada degenerada en una 
agrupación de díadas, lo que no puede de ningún modo dar cuenta del funcionamiento auténticamente triádico de un fenómeno semiótico que sólo puede representarse con una «Y» (ver figura 1).

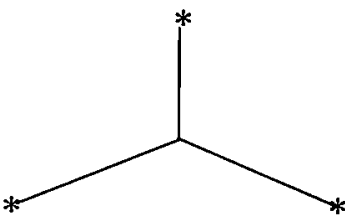

Tríada auténtica

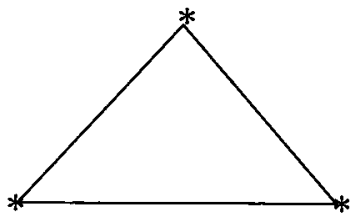

Tríada diádicamente degenerada
*

*

Figura 1

Como, siguiendo a Peirce, una idea no puede comunicarse sin un icono, es preciso utilizar un diagrama correcto para comunicar la tríada que está en el centro de su pensamiento.

\section{La semiótica de Peirce es una semiótica pragmática}

La definición del signo incorporando el interpretante garantiza que la teoría toma a su cargo el efecto del signo sobre una persona, pero no el efecto psicológico sino el efecto sobre esta persona considerada como un ser social, es decir un ser determinado por sus experiencias en un mundo históricamente fechado. Todos los elementos destacados en la definición están «en contexto»:

- Un signo funciona como signo en determinadas circunstancias (como decía $\mathrm{S}$. Freud, presentándose a sus oyentes con un cigarro en la boca: «hay circunstancias en las cuales un cigarro es solamente un cigarro!»).

- Su objeto puede analizarse de tal manera que dé cuenta de su existencia anterior que determinó la institución de su relación aquí y ahora con el signo: se distingue el objeto inmediato, que es el objeto tal como el signo lo representa, y el objeto dinámico, que es la realidad que, de una manera u otra, determinó el signo a su representación. 
- El interpretante que puede analizarse desde tres puntos de vista en interpretante inmediato, que es el sentimiento que el signo produce, interpretante dinámico, que son los actos, en el mundo exterior o en el mundo interior del sujeto, producidos en reacción a la percepción del signo, y el interpretante final, que es el «habitus» al cual estos actos pueden referirse de manera que son considerados como instancias de dichos «habitus» (o leyes).

Así se puede definir un signo con seis elementos formales y determinaciones internas según las cuales el objeto dinámico determina el objeto inmediato, el cual determina el signo, el cual determina el interpretante inmediato, el cual determina el interpretante dinámico, el cual determina el interpretante final. Llamamos «hexádico» este signo considerando que, «olvidando» a los intermediarios encontramos de nuevo el signo triádico, tal como lo hemos definido, tomando como objeto el dinámico y como interpretante el final. Los esquemas de las determinaciones internas en estos casos dos niveles de análisis del fenómeno semiótico son los de la figura 2.

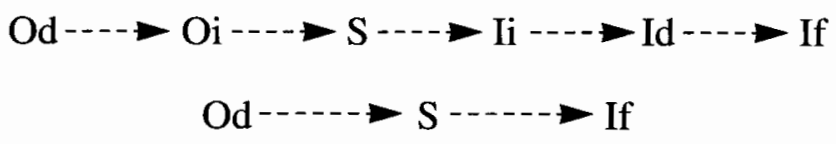

Figura 2

\section{La semiosis}

Ahora podemos incluir en el conjunto de las características esenciales de un signo, de una manera que se puede calificar de «natural», esta observación según la cual el interpretante del signo triádico, determinado por dos relaciones orientadas sucesivas (primeramente la relación del objeto hacia el signo, segundo la relación del signo con el interpretante) puede, a su vez, ser considerado como otro signo del mismo objeto siendo inmediatamente determinado por él. Entonces este signo puede determinar en la misma mente un nuevo interpretante que, a su vez, se convierte en signo y así sucesivamente, «ad infinitum». Vemos entonces que las características ya seleccionadas conducen a tomar en cuenta un proceso formal necesariamente vinculado al fenómeno de interpretación de un signo. Este proceso lo llamamos «semiosis»; aunque sea formalmente infinito, es un proceso que es pragmáticamente interrumpido. 
El esquema del signo triádico de la figura 3 da cuenta de todas las características y consideraciones subsecuentes que hemos hecho hasta aquí.

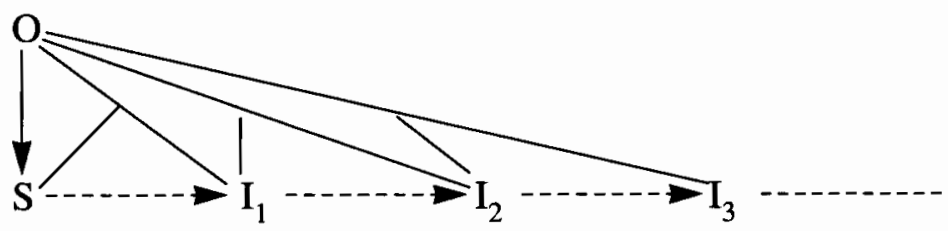

Figura 3

\section{El viaje de la forma}

Sin embargo nos quedamos con una pregunta a la cual tenemos que dar respuesta: $i$ en qué consisten estas determinaciones internas? Un texto de Peirce de 1906 nos ayudará y nos permitirá establecer una definición sintética que nos parece la más adecuada que se puede elaborar. Este texto, sacado de una carta de Lady Welby del 9 de marzo de 1906 es el siguiente:

Utilizo la palabra «signo» en el más amplio sentido de medio para la comunicación de una forma (o figura); siendo medio, es determinado por alguna cosa, 1lamada su objeto, y determina alguna cosa, llamada su interpretante.

Ya vemos que podremos describir dichas determinaciones y relaciones en términos de cierta forma que se encontrará al principio en el objeto y al final en la mente que interpreta el signo. El proceso de la semiosis podremos entonces describirlo como el «viaje» o los «avatares» de una forma depositada en el objeto por la cultura, que sería transferida a la mente por medio del signo. Está claro que no hay transferencia física, lo que significa que la forma de la cual se trata se encuentra potencialmente tanto en el objeto como en el signo y en la mente del intérprete.

\section{6. ¿Cuál es esta forma?}

- Es una pura forma de relaciones, de la cual la fenomenología peirceana nos permite dar una descripción suficientemente precisa por 
las necesidades del análisis: la conciencia de cualquier objeto es un «montón» de «cualidades del sentimiento» (qualities of feeling) organizadas en configuraciones específicas por una familia de relaciones $n$-ádicas (cada una de estas relaciones une exactamente $n$ de esas cualidades). Se puede identificar una relación $n$-ádica con un predicado formal, es decir un $n$-tuple $(-,-,, \ldots,-,-)$ de $n$ sitios que pueden ser ocupados por $n$ cualidades que son fundidas en una nueva cualidad. Estos predicados son combinados por medio de las cualidades comunes, cuando el caso ocurre (Marty 1990).

- Admitiendo este carácter exclusivamente relacional de la conciencia de los objetos del mundo, es evidente que el modo de ser de un objeto consiste en su capacidad de relacionar en la consciencia de cualquier sujeto su propia forma con las formas de los demás objetos.

Entonces, representar un objeto es utilizar otro objeto cuya forma es conveniente (es decir que su propia forma puede relacionarse con la forma de dicho objeto) y que está conectado con él de tal manera que la percepción del segundo produce la presencia en la mente del primero. Esta conexión tiene que ser válida para todos los miembros de una comunidad, porque dentro de una comunidad es patente que comunican los individuos usando signos. Entonces será un «habitus» colectivo, que resulta de un aprendizaje social (en el más amplio sentido de este concepto), es decir una institución social como se concibe en la teoría sociológica del análisis institucional.

\section{El fundamento del signo triádico}

Recapitulando las características seleccionadas junto a la noción de forma transmitida, llegamos a la modelización siguiente en cuanto a los fenómenos semióticos:

- Cada objeto del mundo tiene su propia forma de relaciones que adquiere mediante percepción y «habitus» colectivo por los miembros de una comunidad.

- Representar un objeto es relacionar las formas de cada uno; entonces el representante (el signo) tiene que tener capacidad formal para representar al representado y la relación tiene que ser «ya aquí» para los protagonistas de la comunicación. 
- En consecuencia, hay en la forma del signo una sub-forma $F$ que también está en el objeto y que también está en la mente interpretante por medio de la percepción del signo.

- En la mente esta forma es extendida por medio de inferencias sucesivas hechas en el contexto hasta una forma final (cuando esta forma final es precisamente la forma del objeto, se dice que ha salido bien la comunicación). Esta fase dinámica es la llamada $s e-$ miosis.

- De esta manera se establece entre objeto, signo e interpretante una relación triádica cuyo fundamento (que identificamos al ground de Peirce) es la dicha forma F. Esto está representado en la figura 4.

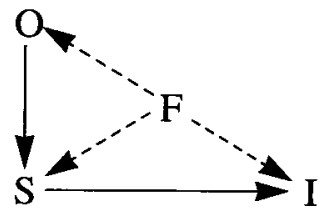

Figura 4

\section{Fenomenología}

Hemos implícitamente admitido hasta aquí que la presencia de un objeto en la mente es correlativa a la formación en esta mente de cierta forma de relaciones específicas de este objeto (ya sea porque el objeto es percibido en el acto o porque ha sido llamado en la mente por haber sido memorizado después de experiencias anteriores).

Entonces, sabiendo que el fanerón (o fenómeno) peirceano es «la totalidad colectiva de todo lo que está presente en la mente» podemos identificarlo con una configuración de formas de relaciones de objetos relacionadas de tal modo que forman una estructura conexa (una totalidad colectiva formal).

En su estado actual, las neurociencias permiten pensar que las formas de las cuales se trata serían realizadas en el cerebro por medio de la activación de ciertas «asambleas de neuronas» (Changeux) características de cada objeto y formadas en el transcurso de la experiencia de estos objetos. 
En consecuencia, describir la fenomenología de los objetos, y más allá de totalidades colectivas de objetos, es describir todas las formas posibles de relaciones entre las configuraciones de las cualidades básicas del sentimiento.

Ahora bien, para afrontar esta extraordinaria complejidad tenemos afortunadamente un teorema (dicho de «reducción triádica») según el cual cada relación $n$-ádica puede construirse combinando de un modo especial, semejante a las combinaciones químicas (productos relativos), relaciones monádicas, diádicas y triádicas, exclusivamente.

Concluimos sobre la fenomenología diciendo que estos tres tipos de formas de relaciones (llamados elementales) constituyen el fundamento formal de las categorías de los elementos de los fenómenos.

\section{Las categorías cenopitagoreanas o faneroscópicas de Peirce}

Recordemos las definiciones que da Peirce de sus categorías (CP, 8328):

- la primeridad es el modo de ser de lo que es tal como es, positivamente y sin referencia a cualquier otra cosa.

- la segundidad es el modo de ser de lo que es tal como es, relativamente a un segundo, pero sin consideración de un tercero, cualquiera que sea.

- la terceridad es el modo de ser de lo que es tal como es, poniendo en relación recíproca un segundo y un tercero.

Entonces consideramos en nuestro modelo formalizado que:

- La primeridad es la parte monádica de la forma de los objetos presentes en la mente, es decir la forma de las cualidades del sentimiento.

- La segundidad es la parte diádica de la forma de los objetos presentes en la mente en forma de parejas de cualidades del sentimiento, es decir la forma de los hechos y de los existentes (un existente puede ser considerado como un hecho definiendo la existencia como oposición de un objeto a todos los demás). 
- La terceridad es la parte triádica de la forma de los objetos presentes en la mente en forma de triples de cualidades del sentimiento, es decir la forma de los conceptos, de las leyes y de los hábitos.

\section{Taxinomías de la representación de un objeto por otro objeto}

La figura 5 muestra todas las posibilidades formales de representar un objeto elemental (es decir para nosotros una forma de relaciones elemental) por un otro objeto. El modo de ser correspondiente, que es el modo de ser del objeto del signo relativamente al dicho signo es indicado en la tercera columna y sirve de definición por las formas degeneradas de las categorías, lo que corresponde rigurosamente a las concepciones de Peirce.

\begin{tabular}{|c|c|c|}
\hline $\begin{array}{c}\text { Forma del objeto } \\
\text { (representado) }\end{array}$ & $\begin{array}{c}\text { Forma del signo } \\
\text { (representante) }\end{array}$ & Modo de ser \\
\hline & & $\begin{array}{c}\text { Terceridad } \\
\text { Auténtica }\end{array}$ \\
\hline & & $\begin{array}{c}\text { Terceridad } \\
\text { degenerada } \\
\text { al 1.er grado }\end{array}$ \\
\hline & $\begin{array}{c}\text { Terceridad } \\
\text { degenerada } \\
\text { al 2. } .^{\text {grado }}\end{array}$ \\
\hline$* * * * * *$ & $\begin{array}{c}\text { Segundidad } \\
\text { Auténtica }\end{array}$ \\
\hline & & $\begin{array}{c}\text { Segundidad } \\
\text { degenerada }\end{array}$ \\
\hline$*$ & & Primeridad \\
\hline
\end{tabular}

Figura 5 


\section{Estructura de orden sobre las categorías faneroscópicas}

La definición de las categorías faneroscópicas en los términos del álgebra relacional tiene consecuencias formales que interpretamos en el marco de la fenomenología. En efecto una tríada presupone tres díadas y tres mónadas porque lo que une a tres cosas une «a fortiori» dos y lo que une a dos une a una.

Entonces, mirando las líneas del cuadro de la figura 5 podemos ver que se puede pasar de ciertas líneas a otras mediante correspondencias adecuadas del mismo tipo que la que está diagramatizada en la figura 6 entre la terceridad degenerada al primer grado y la segundidad auténtica.
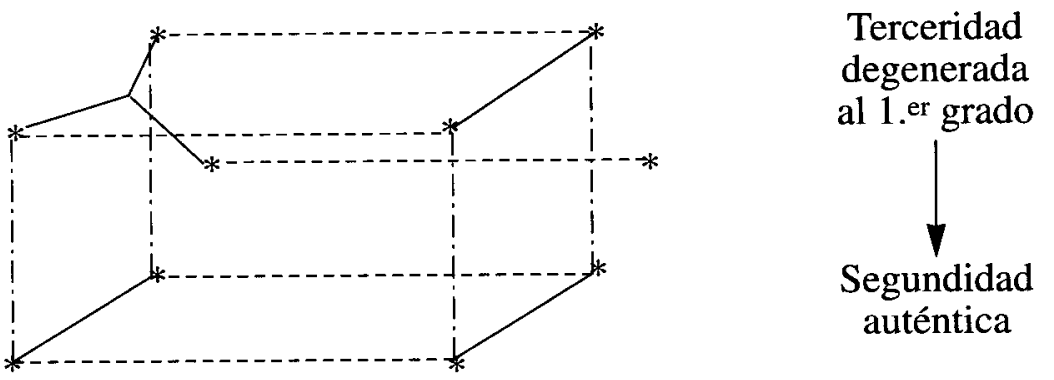

Figura 6

Este formalismo es una manera de hacerse cargo de que, por ejemplo, un concepto es una clase de existentes o una clase de hechos y que una relación del concepto con cualquier otra cosa (otros conceptos, otros existentes o hechos, otras cualidades) se repercute en relaciones entre los existentes o los hechos que están «bajo el concepto», según la expresión de Frege, y esta cosa. Al fin y al cabo de esta investigación obtenemos una estructura algebraica de reticulado (lattice en inglés, treillis en francés) representada en la figura 7.

\section{Las clases de signos}

La figura 5 nos presenta cómo dos elementos primitivos de los fanerones pueden conectarse. Ahora podemos conectar a continuación el signo 


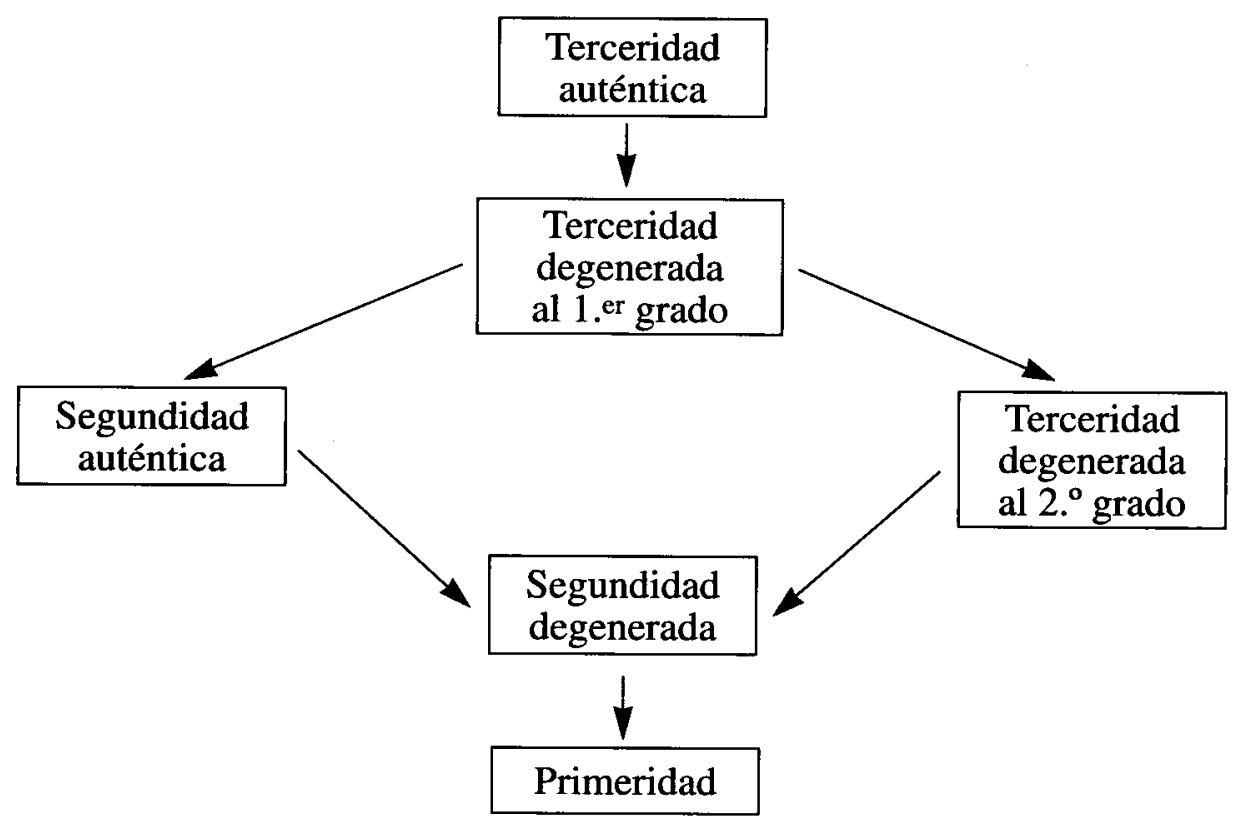

Figura 7

con la mente interpretante con la cual está relacionado de modo que depende de su propia forma. En otras palabras, tenemos que ver las posibilidades de poner una detrás de otra las líneas de la figura 5, manera de describir todos los «viajes» posibles de las formas elementales desde el objeto hasta la mente. Constatamos exactamente diez posibilidades que corresponden cada una a las diez clases de signos anunciadas y descritas por Peirce y formalizan sus definiciones de estas clases (ver 2-254 hasta 2-263). En la figura 8 hemos indicado en cada línea las transformaciones sucesivas de las formas elementales y en la última columna el nombre atribuido por Peirce a cada una de estas clases.

\section{El reticulado de las clases de signos}

El mismo trabajo que hemos hecho sobre las líneas del cuadro de la figura 5 puede hacerse sobre las líneas del cuadro de la figura 8. Obtenemos entonces un nuevo reticulado, llamado reticulado de las clases que muestra las relaciones necesarias de presuposición que existen entre las diez clases de signos. Por ejemplo, entre el símbolo dicente y el legisigno indicial dicente tenemos la relación compleja representada por las líneas punteadas verticales de la figura 9. 


\begin{tabular}{|c|c|c|c|}
\hline Objeto & Signo & Interpretante & Nombre \\
\hline & & & Argumento \\
\hline & & & $\begin{array}{l}\text { Símbolo } \\
\text { dicente }\end{array}$ \\
\hline & & $*$ & $\begin{array}{l}\text { Símbolo } \\
\text { remático }\end{array}$ \\
\hline & * & $*$ & $\begin{array}{l}\text { Legisigno } \\
\text { indicial } \\
\text { dicente }\end{array}$ \\
\hline & * & $\begin{array}{l}* \\
*\end{array}$ & $\begin{array}{l}\text { Legisigno } \\
\text { indicial } \\
\text { remático }\end{array}$ \\
\hline$*$ & & $\begin{array}{l}* \\
*\end{array}$ & $\begin{array}{l}\text { Legisigno } \\
\text { icónico }\end{array}$ \\
\hline$*$ & $*$ & $*$ & $\begin{array}{l}\text { Sinsigno } \\
\text { dicente }\end{array}$ \\
\hline * & * & * & $\begin{array}{l}\text { Sinsigno } \\
\text { indicial } \\
\text { remático }\end{array}$ \\
\hline * & * & * & $\begin{array}{l}\text { Sinsigno } \\
\text { icónico }\end{array}$ \\
\hline$*$ & * & * & Cualisigno \\
\hline
\end{tabular}



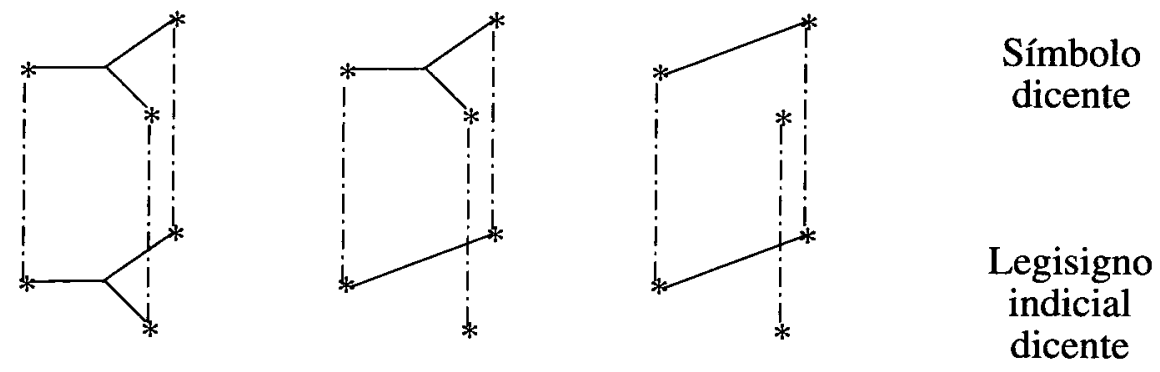

Figura 9

En el reticulado de la figura 10 obtenido al final hemos representado este complejo de relaciones mediante una flecha única.

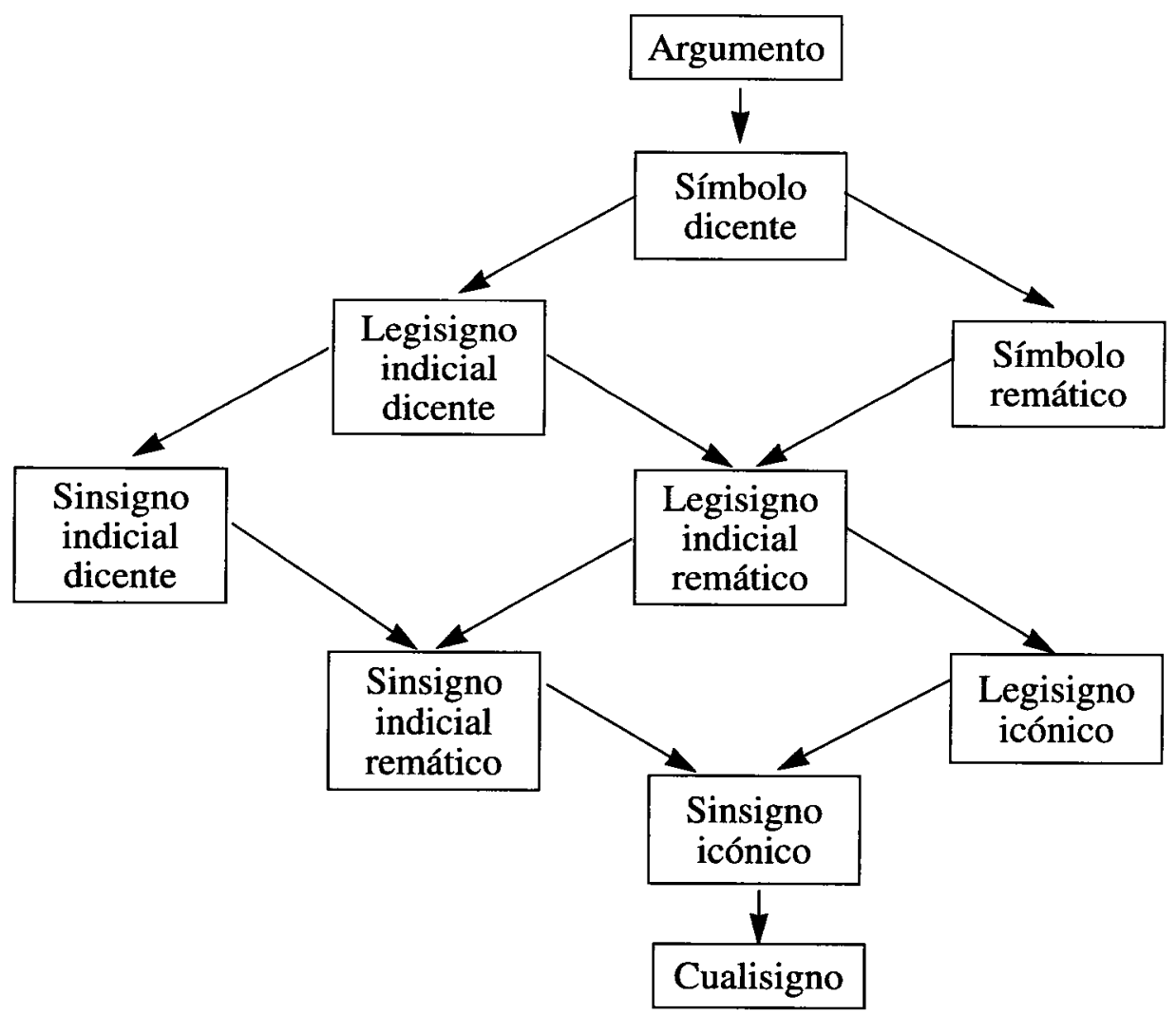

Figura 10 
Este resultado es absolutamente conforme a la intuición de Peirce expresada en 2-264 en términos de afinidades de clases de signos. Sus descripciones de estas clases de 2-254 hasta 2-263 y su dibujo reproducido en la figura 11 ilustran perfectamente las presuposiciones representadas por las flechas del reticulado.

\section{ELEMENTS OF LOGIC}

264. The affinities of the ten classes are exhibited by arranging their designations in the triangular table here shown, which has heavy boundaries between adjacent squares that are appropriated to classes alike in only one respect. All other adjacent squares pertain to classes alike in two respects. Squares not adjacent pertain to classes alike in one respect only, except that each of the three squares of the vertices of the triangle pertains to a class differing in all three respects from the classes to which the squares along the opposite side of the triangle are appropriated. The lightly printed designations are superfluous.

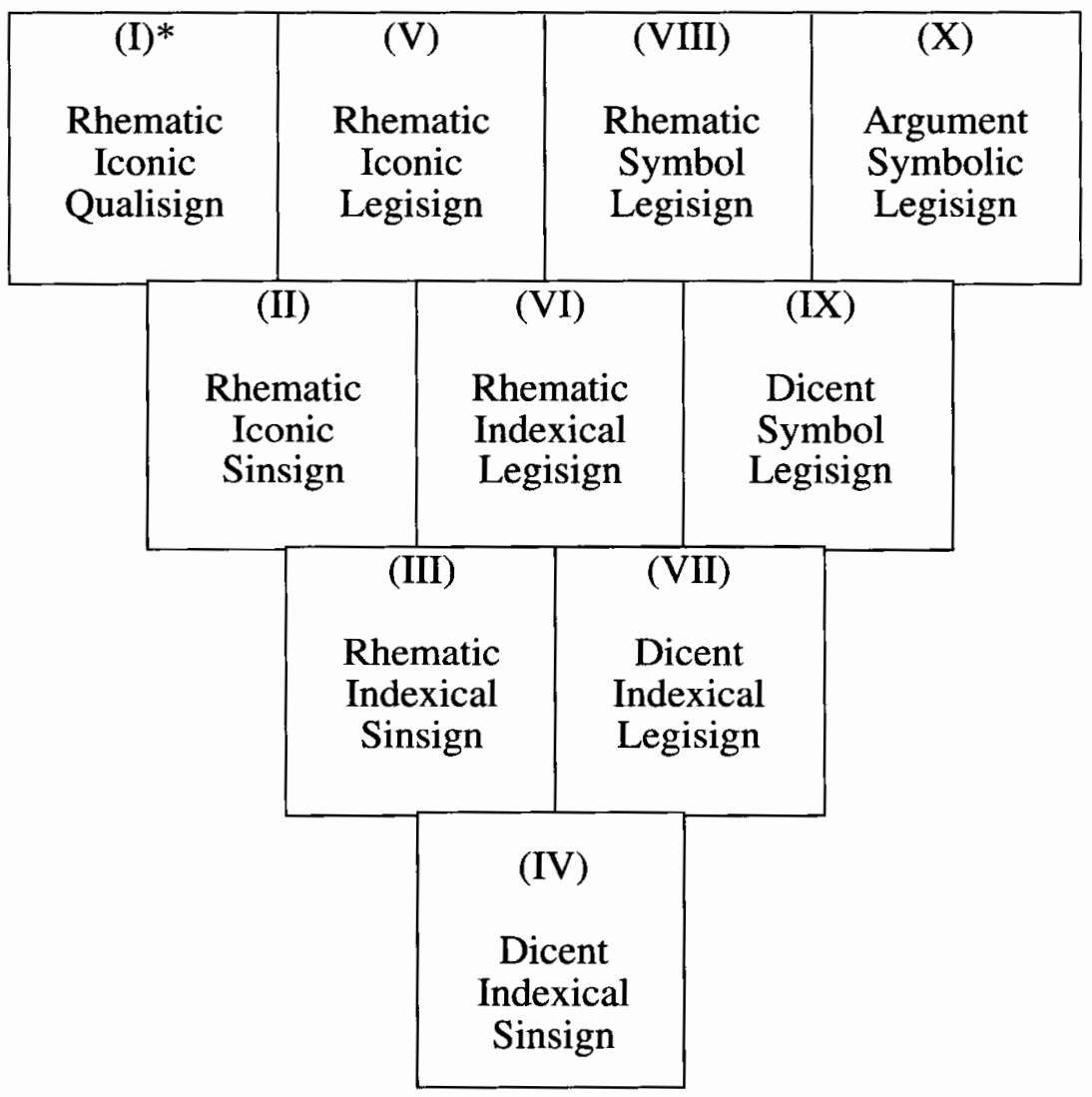

Figura 11 
El mismo tratamiento formal puede ser aplicado a los signos hexádicos; en este caso obtenemos un reticulado de 28 clases de signos (Marty 1990:224).

Ya vemos que estas estructuras inmanentes a las clases de signos nos permitirán salir de las taxinomías tradicionales de los elementos separados de los textos sin posibilidad de alcanzar el nivel propiamente textual. En verdad podemos ahora pensar en la edificación de una verdadera metodología del análisis de los textos.

\section{LA SEMIÓTICA DEL TEXTO}

Avanzaremos en este dominio con dos preocupaciones esenciales:

- En primer lugar, sacar provecho de los resultados muy generales expuestos arriba, considerando un texto como un conjunto organizado de signos como cualquier otro. En otras palabras, empezaremos a elaborar una semiótica específica, sin preocuparnos de las teorías existentes del texto, aplicando la semiótica general peirceana al campo limitado de lo textual. Esto es, evidentemente, un ensayo.

- En segundo lugar, trataremos de ver cómo las nociones clásicas de la lingüística (palabras en general, categorías gramaticales, etc.) y las nociones indiscutibles de la semiótica textual (narrador, actante, personaje, etc.) aparecen cuando las miramos desde nuestro punto de vista fenomenológico y semiótico.

\section{Texto y significación}

Una primera consecuencia de la conceptualización triádica de los fenómenos de significación, dado que incorpora al interpretante, es que el sentido no puede ser inmanente al texto. Sólo puede ser elaborado durante el proceso de la lectura mediante los interpretantes del lector. Para la semiótica peirceana un texto es un conjunto de signos de varias clases según sus estatutos semióticos y cada lector puede construir «a priori» su objeto (el objeto del texto considerado como un signo). Dado que hay, en un primer análisis, tres clases principales de modo de ser de los elemen- 
tos de un texto, tendremos tres niveles «a priori» que serán seis si añadimos las categorías degeneradas. Estos niveles son relacionados por medio de signos linguíísticos especializados en esta función (por ejemplo los deícticos); estos signos constituyen verdaderas pasarelas entre los niveles. En cuanto a la realidad que determinó el texto y al efecto que hace sobre una mente interpretante por medio de los signos lingüísticos podremos distinguir:

- el nivel de las cualidades del sentimiento relacionado con las impresiones producidas por la lectura, nivel esencialmente icónico,

- el nivel de los existentes y de los hechos (la factualidad) relacionado con el mundo real y sus relaciones reales,

-- el nivel de los conceptos, de las leyes, de los hábitos que agrupa a los demás.

\section{El sentido consensual y las coacciones interpretantes}

El consenso constatado la mayoría de las veces relativamente a la significación de un texto puede explicarse mediante las coacciones interpretantes que son signos o configuraciones de signos que, en el texto, dirigen la atención sobre objetos determinados entre todos los objetos posibles; así puede converger la semiosis del texto hacia un objeto final que es casi el mismo para todos los intérpretes miembros de una misma comunidad.

Estos signos son legisignos indiciales, es decir signos que, en virtud de una ley o de un hábito interiorizado por un intérprete, dirigen la atención sobre un objeto (existente, hecho o concepto) con el cual tienen una relación real, es decir independiente de todo intérprete. Los legisignos indiciales remáticos (como los adjetivos y pronombres demostrativos, «este», «éste», «ese», «ése», «aquel», «aquél»..., que están en conexión espacial con otras partes del texto) sólo dirigen la atención sobre el objeto; los legisignos indiciales dicentes traen además una información objetiva sobre él. Por ejemplo, nombres como «simón», «mechero», «sereno», dirigen la atención sobre una época determinada y dicen algo sobre sus modos de locomoción, su modo de alumbrado, la organización de su seguridad. El título de una novela, un prefacio, la especialización de una colección o de un editor son guías que apremian la construcción del sentido hacia el objeto compuesto más o menos deseado por el autor (intencionalidad). Se puede observar que, por el contrario, ciertos autores no 
ponen apremios o los reducen al mínimo para dejar más libertad interpretativa al lector (noción de «obra abierta»).

\section{El texto hojaldrado}

Podemos distinguir ahora, refiriéndose a un interpretante consensual, las palabras del texto según sus funciones semióticas reducidas a sus modos de relación con sus objetos, puesto que las diferencias posibles de interpretación según los intérpretes son así igualadas. Entonces, colocando encima del texto los diferentes objetos en unos planos que representan las categorías faneroscópicas, obtendremos un hojaldrado que es el resultado del encuentro de la expresión escrita o verbal con la semiótica peirceana. Un ejemplo sencillo, construido, por razones didácticas, a partir de una red semántica proveniente de estudios en inteligencia artificial, dará una idea de lo que puede ser el hojaldrado de un texto. El texto es el siguiente:

Clyde es un elefante real. Como todos los elefantes prefiere más los coches que las motocicletas. Pero los elefantes no prefieren más los coches compactos que la Harley de Fred.

Obtenemos el hojaldrado de la figura 12.

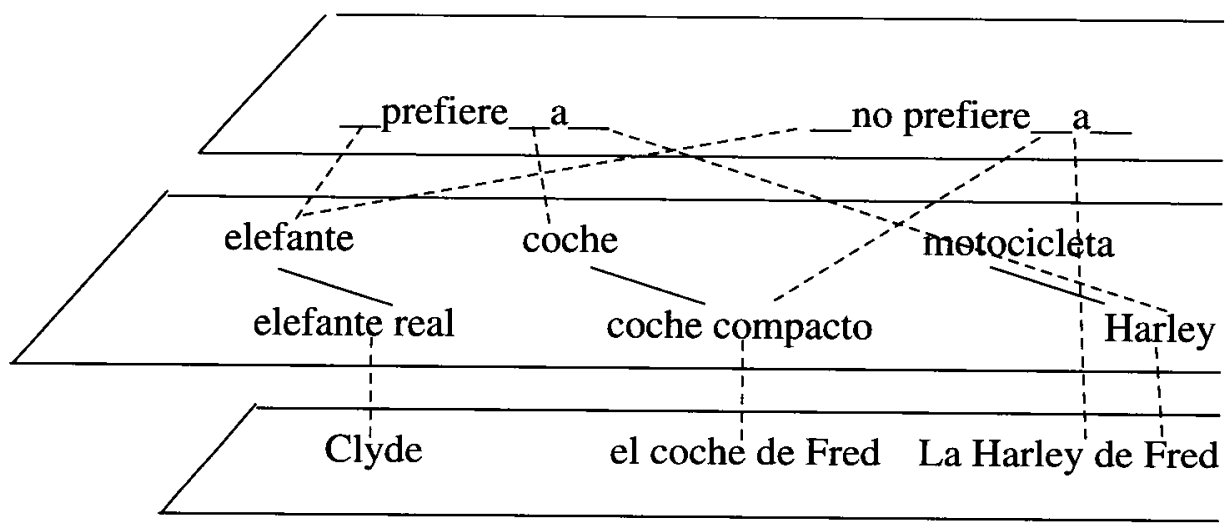

Figura 12 
En los tres planos encontramos de abajo a arriba:

- los seres o las cosas singulares,

- los conceptos entre los cuales unos son relacionados por la relación concepto-subconcepto,

- los predicados (en este ejemplo trivalentes) que combinan los conceptos y/o los seres y las cosas de los planos inferiores. los.

Las líneas punteadas indican los puestos en los cuales hay que poner-

Cambiando el modo de representación podemos introducir elementos implícitos (pero nos parece que intervienen en el proceso de la significación) como ciertas cualidades («blancura», «riqueza», «brillante») y ciertos predicados «-es inherente a-», «-es un-», «-posee-», «-ama a-»,...). Obtenemos así un hojaldrado de cinco hojas correspondiente a las cinco columnas de la figura 13 que son de la izquierda a la derecha: cualidades, seres o cosas, conceptos, predicados bivalentes, predicados trivalentes.

\begin{tabular}{|c|c|c|c|c|c|c|c|c|c|}
\hline \multirow[t]{2}{*}{$\begin{array}{l}\text { Cuali- } \\
\text { dades }\end{array}$} & \multirow[t]{2}{*}{$\begin{array}{l}\text { exis- } \\
\text { tentes }\end{array}$} & \multirow[t]{2}{*}{ conceptos } & \multicolumn{5}{|c|}{$\begin{array}{l}\text { predicados } \\
\text { bivalentes }\end{array}$} & \multicolumn{2}{|c|}{$\begin{array}{l}\text { predicados } \\
\text { trivalentes }\end{array}$} \\
\hline & & & A & B & $\mathrm{C}$ & D & $\mathrm{E}$ & $\mathbf{F}$ & G \\
\hline $\begin{array}{l}\text { blancura } \\
\text { riqueza-- } \\
\text { brillante }\end{array}$ & $\begin{array}{l}\text { Clyde - } \\
\text { Fred - } \\
\text { coche* } \\
\text { moto* }\end{array}$ & $\begin{array}{l}\text { Elef. real } \\
\text { Elefante } \\
\text { Coche comp } \\
\text { Coche } \\
\text { Harley } \\
\text { Moto }-. .\end{array}$ & & ${ }_{-1}^{1}$ & & 1 & - & - & $\int_{-2}^{3}$ \\
\hline NB: & $\begin{array}{l}l \text { asterisc } \\
=1 \text { es in } \\
\text { referido }\end{array}$ & $\begin{array}{l}\text { indica el coc } \\
\text { rente a } 2 ; \mathrm{B}= \\
\text { or los elefant }\end{array}$ & & & & & & 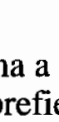 & $\begin{array}{l}=1 \mathrm{e} \\
\text { a } 3\end{array}$ \\
\hline
\end{tabular}


Las líneas horizontales combinadas con las cifras indican en qué puesto de los predicados hay que poner los elementos de la primera columna. Se ve también que algunos predicados bivalentes son incorporados (por degeneración categorial) en predicados trivalentes.

\section{Metodología}

No es necesario recurrir en todas las circunstancias al sentido consensual. En efecto se puede «hojear» un texto analizando los signos producidos por un intérprete durante su lectura y después relacionar las clases de signos obtenidas con las flechas del reticulado de las clases de signos (figura 10). Obtenemos entonces hojaldrados de diez hojas máximo cuando tomamos en cuenta elementos implícitos, cada hoja correspondiente a una clase de signos; en el caso contrario obtenemos solamente un máximo de seis hojas, cada una correspondiente a un legisigno. La metodología utilizada puede describirse en tres fases:

\subsection{Análisis semiótica del texto}

- Inventario de las partes del texto que participan como unidades en el fenómeno semiótico.

- Clasificación de cada una según su clase de signo (el interpretante puede ser particular o consensual, la metodología es la misma).

\subsection{Construcción de las «hojas» encima del texto}

Las clases de signos encontradas en 4.1, una vez colocadas en el reticulado y relacionadas por las flechas del mismo, constituyen el hojaldrado asociado al texto.

\subsection{Determinación de la significación global del texto}

Las propiedades algebraicas del reticulado permiten definir una clase de signos única que es la «suma» (en un sentido generalizado) del conjunto de los signos seleccionados y relacionado por las flechas (un diagrama algebraico). A esta clase-suma damos el papel de «clase de la sig- 
nificación global del texto» porque es la única clase que incorpora formalmente todos los signos que contribuyen a la significación. Es entonces suficiente determinar un signo de esta clase que posea esta propiedad.

De modo simétrico podemos definir un signo que corresponde a la cualidad «sui-generis» del texto («talidad», suchness) es decir la cualidad del sentimiento producida por su lectura.

\section{Aplicación}

Aplicamos esta metodología a un poema titulado «Matemáticas» de Jules Supervielle sacado de Gravitations (traducción libre del autor). El recorte de la primera fase es indicado con las mismas cifras entre paréntesis al principio y al final de cada parte.

(1) Cuarenta niños en una sala Una pizarra negra y su triángulo (1)

(2) Un gran círculo vacilante y sordo Su centro toca como un tambor (2)

(3) Letras sin palabras ni patria En una espera lastimada (3)

(4) El parapeto duro de un trapecio (4)

(5) Una voz se levanta y se calma (5)

(6) Y el problema furioso Se retorna y se muerde la cola (6)

(7) La mandíbula de un ángulo se abre ¿Es una perra, es una loba? (7)

(8) Y todas las cifras de la tierra Todos estos insectos que deshacen Y que rehacen su hormiguera(8)

(9) Bajo los ojos fijos de los chicos.(9)

Atribuimos a cada parte los objetos siguientes: (1) aula «arquetípica»; (2) círculo vivo; (3) letras humanizadas; (4) parapeto trapezoidal; (5) voz indeterminada; (6) concepto de problema «animalizado»; (7) ángulo 
«animalizado»; (8) cifras «hormigazadas»; (9) niños petrificados. Es claro que estas atribuciones son muy personales y en consecuencia muy discutibles y polémicas. Pero eso importa poco porque no concierne a la metodología.

Construimos ahora el hojaldrado de la figura 14 después de haber determinado las clases de cada signo según su relación con su objeto y con su interpretante.

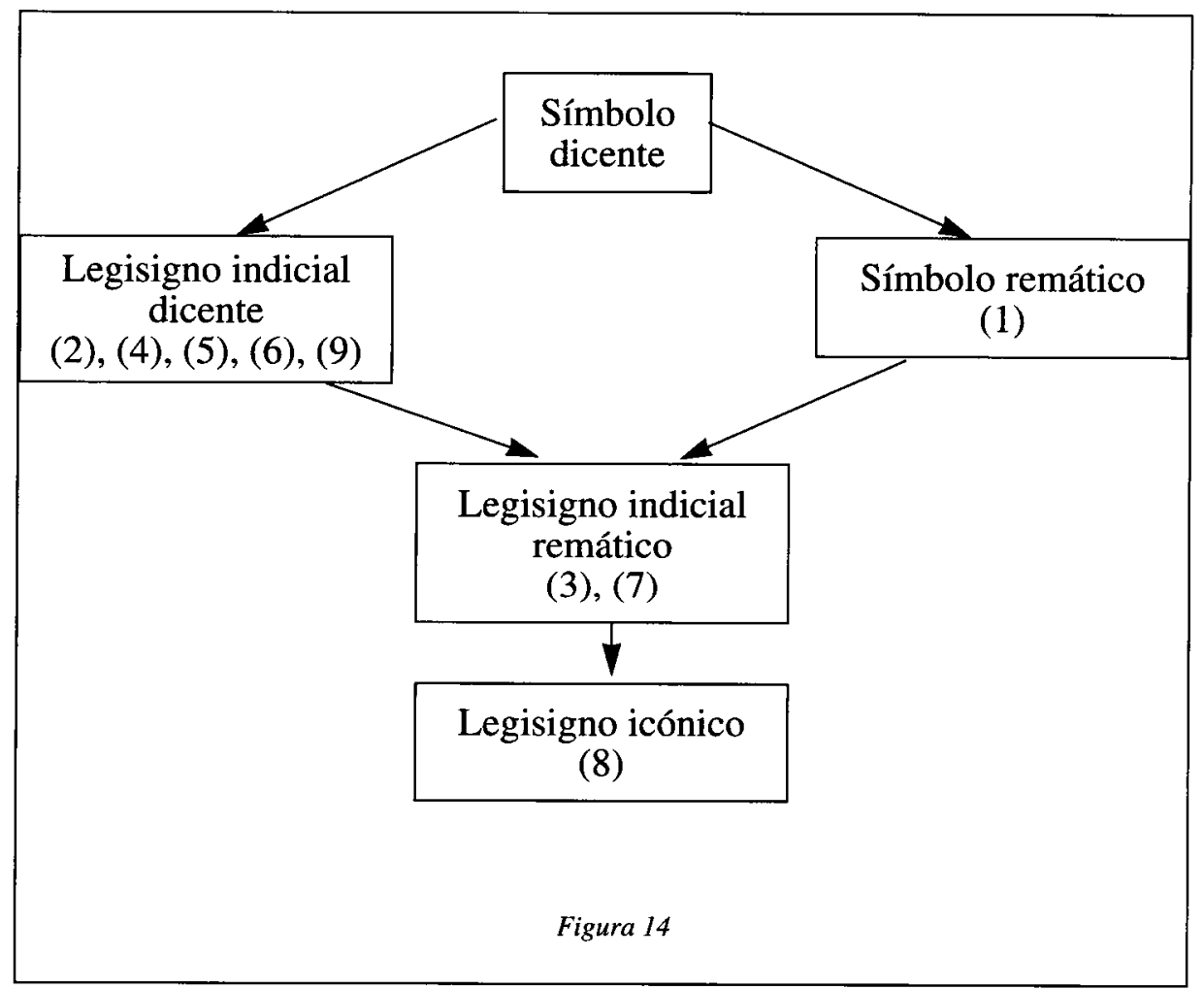

Obtenemos cuatro hojas y vemos que la clase-suma es la clase del símbolo dicente que no figura en la fase anterior. Proponemos la proposición (símbolo dicente) siguiente para globalizar el sentido: «La matemática es un monstruo animal». Simétricamente, la «talidad» de este texto sería «la animalidad matemática». El hojaldrado del poema muestra (es un icono) la estructura fenomenológica del hundimiento de las matemáticas en el reino animal realizada por Supervielle; un modo poético de crear una teratología. 


\section{Los estatutos semióticos de las palabras}

Todas las palabras son réplicas de legisignos, es decir instancias o casos de convenciones sociales «establecidas por los hombres». Como hay seis tipos de legisignos (es decir seis modos para un interpretante de conectar una palabra con su objeto) cada palabra encontrará, como ya lo hemos visto, su objeto en una de las seis «hojas» encima del texto. Las correspondientes categorías gramaticales, identificadas en su mayor parte por Peirce, son las siguientes:

— los legisignos icónicos: adjetivos cualificativos, adverbios de manera;

- los legisignos indiciales (remáticos o dicentes): nombres propios; pronombres personales, pronombres demostrativos, pronombres relativos, determinantes definidos, adverbios de lugar, adverbios de tiempo (en general todos los morfemas de conexión);

- los símbolos (legisignos simbólicos remáticos, dicentes o argumentales) que representan clases de seres o de cosas (nombres comunes) y relacionales entre estas clases (verbos monovalentes, bivalentes o trivalentes).

\section{Un ejemplo: el nombre propio}

Hemos clasificado anteriormente el nombre propio como legisigno indicial; eso significa que es conectado con un individuo singular (que puede ser un conjunto, pero totalizado, como un país, una familia, etc.) mediante el conocimiento de este individuo que posee el intérprete. Pero el reticulado de las clases de signos nos muestra que cada legisigno indicial contiene necesariamente (porque los presupone lógicamente) legisignos icónicos. Por eso puede producir en la mente interpretante sensaciones y pensamientos que el individuo designado produce y que produce también una clase particular de individuos (es lo que Barthes llama «hipersemanticidad» del nombre propio). Así «Guermantes» (Proust) evoca inmediatamente la aristocracia, «Monsieur de Sotenville» (Molière) la burguesía presumida, «Sancho Panza» la clase popular, etc. El legisigno indicial contiene también cualisignos; algunos de ellos (por ejemplo, fonéticos) pueden ser seleccionados como símbolos, como en «Rastignac» (Balzac). 


\section{El estatuto semiótico del narrador}

En la narratoria de A. J. Greimas el narrador y el enunciador son actantes de la comunicación, pero mientras que el enunciador es el productor implícito y presupuesto de la comunicación o de la enunciación, el narrador es explícitamente instalado en el discurso-enunciado en forma de pronombre «yo». Este narrador es un simulacro del enunciador y no puede ser confundido con él. Su instalación en el discurso-enunciado presupone un proceso de desembrague seguido de un embrague a fines de producir la enunciación-enunciada (un simulacro de la enunciación que produce la ilusión enunciativa).

Nos parece que se puede incorporar la noción de narrador a la semiótica peirceana del texto apoyándose en esta característica de «simulacro del enunciador» que podemos traducir en términos de iconismo. El narrador sería, representado en el texto, un interpretante de los existentes y de los hechos de un mundo real (existente o ficticio) del cual se trata en el texto. El narrador considerado así soportaría entonces la ideología y la intencionalidad del autor que por medio de la noción de interpretante último, que según Peirce consiste en un cambio de hábito, permite tomar en cuenta este efecto sobre la mente del lector más o menos deseado por el autor en la comunicación literaria. El papel de narrador pueden desempeñarlo en el texto varias configuraciones de personajes que serían las réplicas del narrador (es decir sinsignos icónicos réplicas de legisignos icónicos). Puede ser completamente ficticio en relatos en «él», un personaje testimonio en relatos en «yo», un personaje actante-sujeto o un narrador «estallado» en varios personajes, siendo cada uno de ellos portador de una parte de los interpretantes (lo que Greimas llama «focalización»).

Estas consideraciones deben considerarse como «pistas» para la investigación que podrían revelarse bastante heurísticas en el porvenir.

\section{CONCLUSIÓN}

Ya vemos que en cuanto a la semiótica peirceana del texto, si la comparamos con las teorías desarrolladas desde hace mucho más tiempo, todo o casi todo queda por hacer. Sin embargo, la tríada lleva una riqueza formal expresada en posibilidades combinatorias que nos parecen «a priori» más adecuadas para soportar la complejidad de los fenómenos calificados de literarios. Es en este campo, entonces, donde se puede inten- 
tar una apuesta intelectual bastante estimulante; como lo escribía Peirce: «en la investigación científica, como en otras empresas, es válido el dicho: «donde no hay riesgo, no hay provecho».

\section{Referencias bibliográficas}

MaRTy, C., MarTY, R. (en prensa): 99 réponses sur la sémiotique. Montpellier: Centre Regional de Documentation Pédagogique.

MARTY, R. (1990): L'Algèbre des signes, Essai de Sémiotique scientifique d'aprés Ch. S. Peirce. Amsterdam-Philadelphie: John Benjamins.

PeIRCE, CH. S. Manuscritos microfilmados. Harvard Library (1931-1958). Collected Papers. Cambridge MA: Harvard University Press.

- (1976): The News Elements of Mathematics. Eisele, C. (ed.). The Hague: Mouton. 